\title{
Diagnostic value of chest CT scanning for determination of Covid-19 severity in individual lung lobes
}

\author{
Witold Krupski ${ }^{1, A, C, E-F \oplus}$, Andrzej Gorecki ${ }^{2, A-C, E-F} \oplus$, Joanna Kruk-Bachonko ${ }^{3, C-F \oplus}$ \\ ${ }^{1}$ Medical University, Lublin, Poland \\ 2 Department of Radiology VOXEL, Łańcut, Poland \\ ${ }^{3}$ University Hospital No 1, Lublin, Poland \\ A - Research concept and design, B - Collection and/or assembly of data, C - Data analysis and interpretation, \\ $D$ - Writing the article, $E$ - Critical revision of the article, $F$ - Final approval of the article
}

Krupski W, Gorecki A, Kruk-Bachonko J. Diagnostic value of chest CT scanning for determination of Covid-19 severity in individual lung lobes. Ann Agric Environ Med. 2022; 29(1): 115-119. doi: 10.26444/aaem/144842

\begin{abstract}
Introduction and Objective. CT examination is an important diagnostic tool in assessment of the severity of the infection and course of the disease. The aim of the study was to evaluate the degree and frequency of involvement of individual lung lobes and the population Lobe Involvement Coefficient (pLIC) value in the investigated population.

Materials and method. The analyzed material comprised 124 patients aged 18-92 years. CT examinations were performed using a 16- and 32-row CT LightSpeed apparatus. The spatial distribution of typical Covid -19 pathological changes was analyzed, divided into five lung lobes. The degree of the severity of lobe involvement was assessed using counters and percentages, as well as the population Lobe Involvement Coefficient (pLIC). Statistical analysis of data was performed with the use of Statistica 10.0 software. Values were measured on an oridinal scale. Anova Friedman's test was used to compare lobes.

Results. Statistically significant differences in the involvement between most of the individual lobes were shown. There was no statistically significant difference in the degree of lobe involvement between the left and right upper lobes, nor in the left and right lower lobes. The highest pILC was demonstrated for the lower lobe and the lowest value was obtained for the middle lobe.

Conclusions. The lower lobes were affected most frequently and most severely, with no statistical difference between the right and left sides. The middle lobe was affected relatively least frequently and lightly. The introduced pLIC index allows quantitative assessment of individual lobes involvement in relation to the entire studied population.
\end{abstract}

Key words

SARS-CoV-2, tomography, Covid-19 diagnostic testing

\section{INTRODUCTION}

Chest CT scan has a very high sensitivity and low specificity in detecting SARS-CoV2 infection $[1,2,3]$. The CT exam with a positive PCR test is an important diagnostic tool in the assessment of the severity of the infection and course of the disease. Most authors show that typical radiological symptoms of Covid-19 infections are ground-glass consolidations, crazy paving, vascular dilatation, traction bronchiectasis, and subpleural bands $[1,2,3]$. Many studies report that SARSCoV2 infection is more common peripherally and bilaterally. Several studies have investigated the relationship between the distribution of the disease symptoms between the upper and lower zones ${ }^{4}$. To our knowledge, there are only a few reports on the distribution of pathological changes in the course of SARS-CoV2 infection in relation to the anatomical division of lung lobes $[5,6]$. Computed tomography perfectly reflects the anatomic boundaries of the lung lobes and facilitates identification of the specific Covid-19 radiological changes in the particular lobes. The lobes of the lungs differ

Address for correspondence: Joanna Kruk-Bachonko, University Hospital No 1 , Lublin, Poland

E-mail: asiakruk1@wp.pl

Received: 08.10.2021; accepted: 12.12.2021; first published: 28.12.2021 anatomically in terms of the course of the bronchi and have varied ventilation [7]. Therefore, we considered it interesting to examine whether the anatomical boundaries of the lungs contribute significantly to the distribution of radiological changes in the course of SARS-CoV 2 infection.

The aim of the study was to evaluate the degree and frequency of involvement of individual lung lobes and the population Lobe Involvement Coefficient ( $\mathrm{p}$-LIC) value in the investigated population.

\section{MATERIALS AND METHOD}

The study population comprised hospital patients in the Łańcut Medical Centre in the Subcarpathian Province of southeast Poland, confirmed to be infected with SARS-CoV2 by positive PCR test. Patients with known coexisting diseases which can present similar imaging abnormalities, including interstitial lung diseases and congestive heart failure, were excluded from the final analysis.

Radiological data were collected from obligatory chest CT examinations of all patients infected with the SARS-CoV2 virus performed at the VOXEL Department of Radiology in the Łańcut Medical Centre. CT scan was performed immediately after diagnosis. 
The analyzed material consisted of 124 patients aged $18-92$ years (mean age: 55.99, SD: 16.99). The study covered 69 men aged $27-89$ years (mean age: 54.86 , SD: 17.14 ) and 55 women aged $18-92$ years (mean age: 67.42 , SD: 16.86 ).

CT examinations of the chest were performed using a GE 16- and 32-row CT LightSpeed apparatus, and with the spiral technique in $2.5 \mathrm{~mm}$ thick axial sections (continuous layers) reconstructed to a thickness of $1.25 \mathrm{~mm}$ (pulmonary reconstruction) with the high-resolution HRCT reconstructive algorithm. Retrospective analysis of each chest CT examination was performed by consensus between two radiologists.

The most common symptom was the ground-glass opacification, which alone accounted for $41.1 \%$, and with consolidation a further $35.5 \%$ of the total number of respondents. In addition, in the examined material frequent symptoms were thickening of the interlobular septa (53.2\%) and crazy paving (41.9\%), as well as areas of consolidation (40.3\%).

In the spatial distribution of typical Covid-19, pathological changes was analyzed in five lung lobes: right upper lobe (RUP), right middle lobe (RML), right lower lobe (RLL), left upper lobe (LUL), and left lower lobe (LLL). The degree of severity of lobe involvement was classified based on a fivepoint scale, following the adapted Radiographic Assessment of Lung Edema score proposed by Warren et al. [8]:

Score 0 - no radiological changes.

Score 1 - radiological changes typical for Covid-19 infections covering up to $25 \%$ of the lobe area.

Sore 2 - radiological changes typical for Covid-19 infections covering $26-50 \%$ of the lobe area.

Sore 3 - radiological changes typical for Covid-19 infections covering $51-75 \%$ of the lobe area.

Score 4 - radiological changes typical for Covid-19 infections covering more than $75 \%$ of the lobe area.

Each lobe was assessed using the score scale from 0-4 points for a single patient and from 0-496 points for the entire study population.

In order to standardize the comparison between individual lobes, the definition of the population Lobe Involvement Coefficient (p-LIC) was introduced, an index invented by authors. This coefficient defines the mean severity of involvement of a particular lobe for the entire study population. P-LIC was calculated according to the formula: sum of points of the severity of particular lobe involvement according to the above-mentioned five-point scale/number of analyzed lobes. This showed the average number of points for a given lobe in the studied population.

In an individual assessment of a single patient, a percentage of the involvement of individual lobes or the entire lung parenchyma in general is sufficient. However, when considering the frequency and intensity of a particular lobe involvement in the entire population, it is difficult to assess the percentage of individual lobe involvement in the entire study population. On the other hand, it seems that the p-ILC coefficient can be useful in such an analysis and allow for an objective and repeatable assessment, both in the initial diagnosis and, for example, when monitoring the treatment of a given population.

Statistical analysis. Performed with the use of Statistica 10.0 software. The values of the parameters measured - measured on an ordinal scale - were characterized using the number and percentage. Anova Friedman's test was used to compare the five individual lobes assessed in each subject according to the ordinal scale of the measurement. After the differences were found to be significant, Dunn's post hoc test was used to compare the individual pairs of lobes. A $5 \%$ inference error was assumed, and therefore a significance level of $p<0.05$ indicating the existence of statistically significant differences [9]

\section{RESULTS}

The degree of the severity of lobe involvement was classified in a five-point scale described in detail in the Materials and Method section.

Table 1 presents the cumulative number of points obtained for individual lobes in the entire examined population and the calculated population Lobe Involvement Coefficient (p-LIC).

Table 1. Cumulative number of points obtained for individual lobes in the entire study population and the calculated population Lobe Involvement Coefficient (pLIC)

\begin{tabular}{lccccc}
\hline & $\begin{array}{c}\text { Right } \\
\text { Upper } \\
\text { Lobe }\end{array}$ & $\begin{array}{c}\text { Left } \\
\text { Upper } \\
\text { Lobe }\end{array}$ & $\begin{array}{c}\text { Right } \\
\text { Middle } \\
\text { Lobe }\end{array}$ & $\begin{array}{c}\text { Right } \\
\text { Lower } \\
\text { Lobe }\end{array}$ & $\begin{array}{c}\text { Left } \\
\text { Lower } \\
\text { Lobe }\end{array}$ \\
\hline $\begin{array}{l}\text { Sum of points for a } \\
\text { given lobe } \\
\text { in the five-point scale } \\
\text { of lobe involvement }\end{array}$ & 121 & 128 & 99 & 170 & 167 \\
\hline $\begin{array}{l}\text { Population Lobe } \\
\text { Involvement } \\
\text { Coefficient (pLIC) }\end{array}$ & 0.976 & 1.032 & 0.798 & 1.371 & 1.347 \\
\hline
\end{tabular}

Figure 1 presents the chest CT of a patient with COVID infection with a relatively uniform involvement of both the lower and upper lobes, and relative saving of the middle lobe.

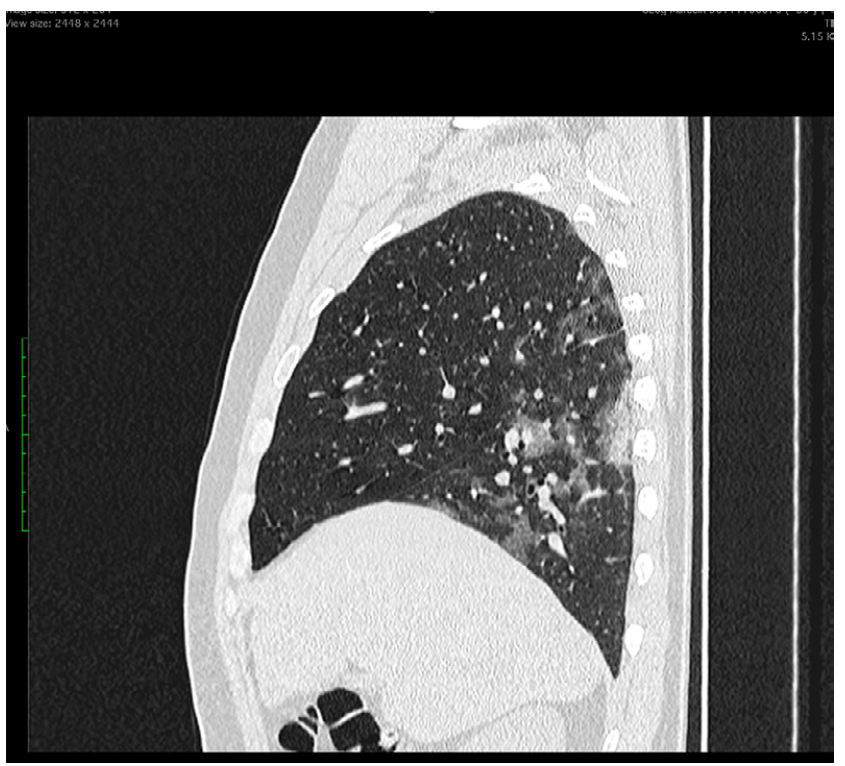

Figure 1a. Chest CT in sagittal reformat of the right lobe of a patient with COVID infection. Figure presents relatively uniform involvement of both the lower and upper lobes and relative saving of the middle lobe 


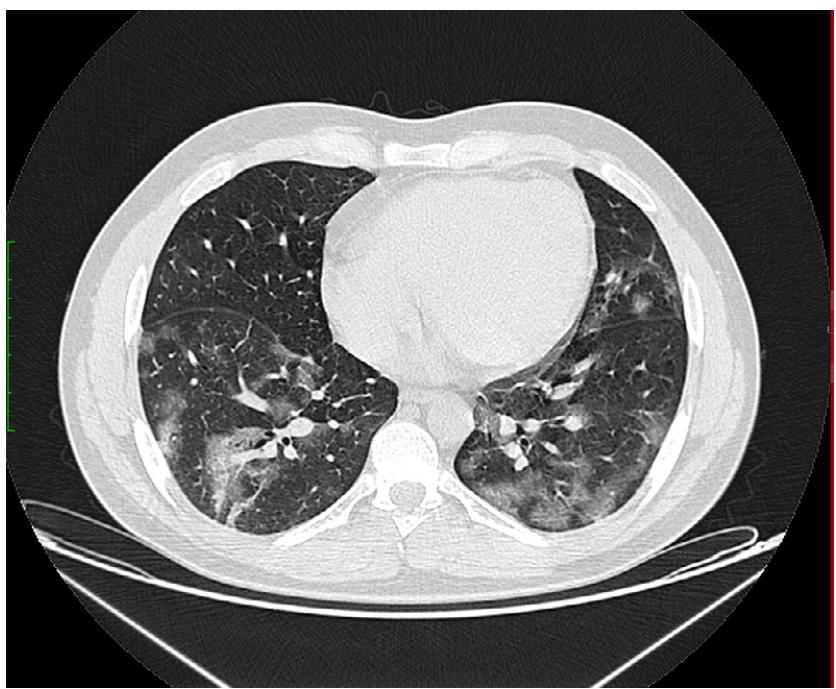

Figure 1b. Chest $\mathrm{CT}$ in axial view at the level of the middle lobe. Figure presents relatively uniform involvement of both the lower and upper lobes and relative saving of the middle lobe

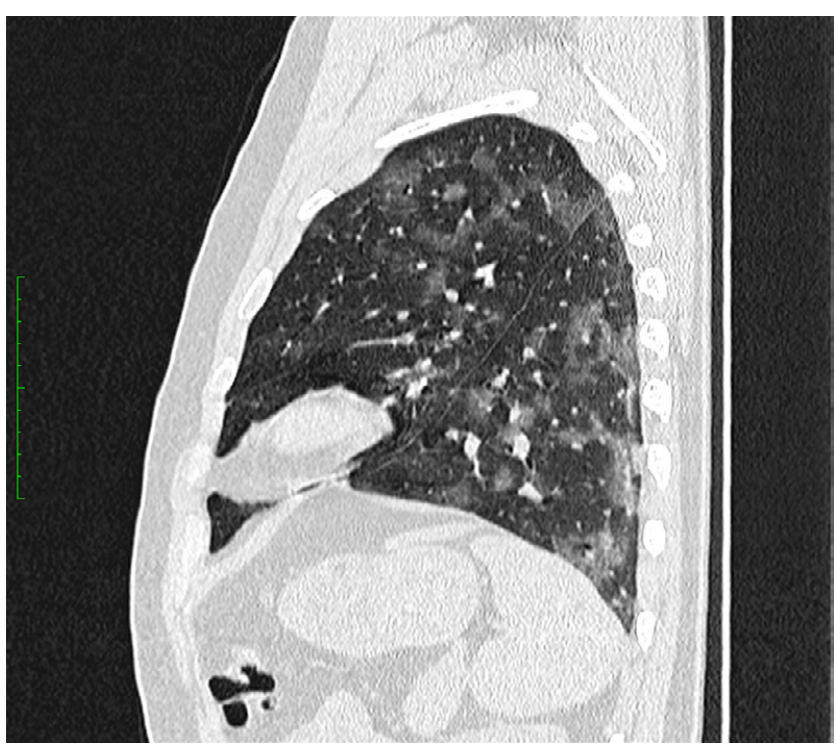

Figure 1c. Chest CT in sagittal reformat of the left lobe of a patient with COVID infection. Figure presents relatively uniform involvement of both the lower and upper lobes and relative saving of the middle lobe

Table 2 presents the severity of involvement of the individual lobes in the entire examined population in a five-point severity scale.
The obtained results revealed a more severe course of infection in the left and right lower lobes of lungs. The middle right lobe exhibited a relatively low degree of destruction compared to the others.

The introduced $\mathrm{p}$-LIC shows these dependencies in relation to a particular lobe. P-LIC defines the mean severity of particular lobe involvement in the entire study population.

Descriptive statistical data of the investigated population are shown in Table 2 and Figure 2.

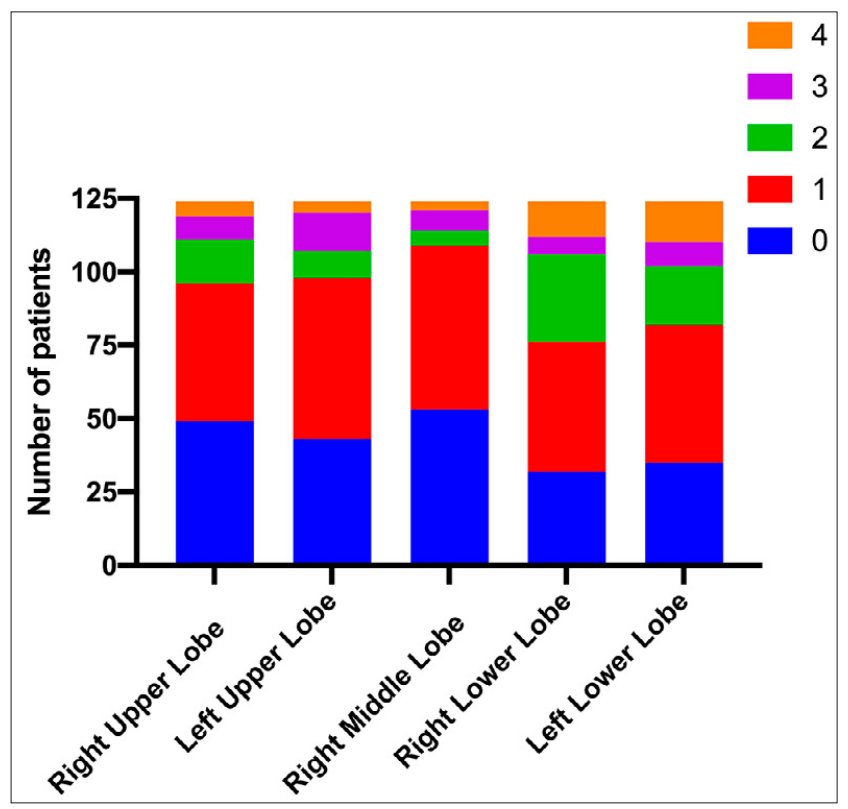

Figure 2. Schematic division of the severity of involvement of individual lobes in the adapted Radiographic Assessment of Lung Edema score scale: Blue bar: score 0 - no radiological changes Red bar: score 1 - radiological changes typical for COVID-19 covering up to $25 \%$ of the lobe area Green bar: score 2 - radiological changes typical for COVID-19 covering $26-50 \%$ of the lobe area Purple bar: score 3 - radiological changes typical for COVID-19 covering 51-75\% of the lobe area Orange bar: score 4 - radiological changes typical for COVID-19 covering more than $75 \%$ of the lobe area

Lobes with a lower p-LIC score were found to show more often a milder course of infection according to the Radiographic Assessment of Lung Edema score scale. Statistically significant differences in the involvement between most of the individual lobes were shown. There was no statistically significant difference in the degree of lobe involvement between the left and right upper lobes, nor the left and right lower lobes (Tab. 3).

Table 2. Severity of the involvement of individual lobes in the entire study population in a five-point severity scale based on the adapted Radiographic Assessment of Lung Edema score: 0 - no radiological changes; 1 - radiological changes typical for COVID-19 covering up to $25 \%$ of the lobe area; 2 - radiological changes typical for COVID-19 covering 26-50\% of the lobe area; 3 - radiological changes typical for COVID-19 covering $51-75 \%$ of the lobe area; 4 - radiological changes typical for COVID-19 covering more than $75 \%$ of the lobe area.

\begin{tabular}{|c|c|c|c|c|c|c|c|c|c|c|}
\hline \multirow[b]{2}{*}{$\begin{array}{l}\text { Severity of the lobe } \\
\text { involvement }\end{array}$} & \multicolumn{2}{|c|}{ Right Upper Lobe } & \multicolumn{2}{|c|}{ Left Upper Lobe } & \multicolumn{2}{|c|}{ Right Middle Lobe } & \multicolumn{2}{|c|}{ Right Lower Lobe } & \multicolumn{2}{|c|}{ Left Lower Lobe } \\
\hline & $\begin{array}{l}\text { Number of } \\
\text { lobes }\end{array}$ & $\%$ & $\begin{array}{l}\text { Number of } \\
\text { lobes }\end{array}$ & $\%$ & $\begin{array}{l}\text { Number of } \\
\text { lobes }\end{array}$ & $\%$ & $\begin{array}{l}\text { Number of } \\
\text { lobes }\end{array}$ & $\%$ & $\begin{array}{l}\text { Number of } \\
\text { lobes }\end{array}$ & $\%$ \\
\hline 1 & 47 & 37.9 & 55 & 44.4 & 56 & 45.2 & 44 & 35.5 & 47 & 37.9 \\
\hline 2 & 15 & 12.1 & 9 & 7.3 & 5 & 4.0 & 30 & 24.2 & 20 & 16.1 \\
\hline 3 & 8 & 6.5 & 13 & 10.5 & 7 & 5.6 & 6 & 4.8 & 8 & 6.5 \\
\hline 4 & 5 & 4.0 & 4 & 3.2 & 3 & 2.4 & 12 & 9.7 & 14 & 11.3 \\
\hline
\end{tabular}


Table 3. Statistically significant differences in the COVID-19 infection severity between individual lobes. Wilcoxon multiple comparison analysis

\begin{tabular}{lcc}
\hline Multiple comparison test & p-value & Significant \\
\hline RUL vs. RML & 0.005 & YES \\
\hline RUL vs. RLL & 0.000 & YES \\
\hline RUL vs. LUL & 0.528 & NO \\
\hline RUL vs. LLL & 0.000 & YES \\
\hline RML vs. RLL & 0.000 & YES \\
\hline RML vs. LUL & 0.003 & YES \\
\hline RML vs. LLL & 0.000 & YES \\
\hline RLL vs. LUL & 0.000 & YES \\
\hline RLL vs. LLL & 0.632 & NO \\
\hline LUL vs. LLL & 0.000 & YES
\end{tabular}

RUL - Right upper lobe; RML - right middle lobe; RLL - right lower lobe; LUL - left upper lobe LL - left lower lobe

\section{DISCUSSION}

Computed tomography plays a particularly important role in the diagnosis of patients with viral pneumonia in the course of Covid-19 infection [3, 10]. Chest CT imaging has high sensitivity in diagnosing Covid-19 and can be considered as a full comprehensive assessment, monitoring subsequent lung lesions or evaluating the degree of pulmonary parenchyma involvement $[3,6,10,11]$. Most reports indicate that inflammatory lesions in the lungs in the course of Covid-19 infection are multiple and scattere, with a predilection for the subpleural localization and the dorsal areas of the lungs $[2,3,4,6,11]$. Computed tomography also allows precise anatomical assessment of the lungs with division into individual lobes.

The current study showed statistically significant differences in the involvement between the individual lobes of the lungs. There were no differences in the Covid-19 infection severity, only between the left and right upper lobes and between the left and right lower lobes. This observation indicates that the anatomical differences in the lung structure may have a real impact on the distribution of destruction in the course of SARS-CoV2 infection.

The individual lobes differ in the diameter of the bronchi and their ventilation $[7,12]$; additionally, the force of gravity affects both the ventilation of the airflow, and causes a pressure gradient between the apex and base of the lungs, leading to different expansion of the lobes [7].

Descriptive statistics and p-ILC may be useful for determination of the severity of destruction of the particular lobes in the examined population. The relatively greatest advancement of changes was indicated in the lower lobes, with the values of the p-LIC index of 1.347 and 1.371. Detailed analysis of the infection severity for the individual lobes in the five-point scale indicated score 4 of advancement most frequently in the lower lobes.

P-ILC may contribute to the standardization of the radiological assessment of the involvement of the pulmonary lobes in the 2019 coronavirus disease (COVID-19) in terms of the examined population, not just the individual. This may facilitate the assessment of the radiological response in a given patient population and affect the alignment of the therapeutic algorithm
There are reports in the literature on lung ventilation models, which report that the lower lobes have a greater volume and therefore a higher flow rate. Perhaps this determines the greater severity of the involvement of these lobes [7]. Similar results were reported by other authors describing frequent involvement of the lower lobes $[3,5$, $13,14]$.

An interesting observation is the fact that the middle lobe is affected by the disease relatively least severely - pLIC 0.798. A detailed analysis indicated that this lobe was most often assigned involvement severity scores 0 and 1 . The same researchers working on a five-lobe lung ventilation model noticed the lowest frequency of detachment of microfluidic bodies with diameters of $0.01 \mu \mathrm{m}$ and $10 \mu \mathrm{m}$ in the middle lobe ${ }^{7}$.Similar results were shown by other authors, i.e. relatively lighter involvement of the central lobe in the course of Covid-19 [3, 12, 14].

Most original papers and meta-analyses report a similar percentage distribution of the infection severity in individual lobes $[3,5,13,14]$. Nevertheless, it is usually only one of the many aspects assessed without a detailed analysis of this feature. To the best of our knowledge, no statistic significance of the distribution differences has been reported so far. We believe that our work is a valuable supplement to our earlier observations, and that the introduced pILC is a useful quantitative indicator of lobe involvement for the entire study population, not only for individual patients.

This study has several limitations. The first is the size of the group. Similar analyses should be carried out on a larger population to verify the propsed theses. Another limitation is the selection of the group. Our patients were examined in the early stages of infection. Hence, it is not know whether the distribution of the lobe involvement indicated will persist unchanged during the evolution of Covid-19 disease.

\section{CONCLUSIONS}

CT examination can be a very useful tool for assessing severity in the course of SARS-CoV2 infection. The introduced pLIC index allows a quantitative assessment of the involvement of individual lobes in relation to the entire studied population. The lower lobes are affected most frequently and most severely, with no statistical difference between the right and left sides. The middle lobe is affected relatively least frequently and lightly.

Ventilation mechanisms dependent on the anatomical structure of individual lobes of the lungs may contribute significantly to the distribution of changes in the course of Covid-19 disease.

\section{REFERENCES}

1. M-Y Ng, EYP Lee, Yang J, et al. Imaging Profile of the COVID-19 Infection: Radiologic Findings and Literature Review. Radiology Cardiothoracic Imaging. 2020; (2): e200034.

2. Bernheim A, Mei X, Huang M, et al. Chest CT Findings in Coronavirus Disease-19 (COVID-19): Relationship to Duration of Infection. Radiology. 2020; (295): 200463.

3. Chung M, Bernheim A, Mei X, et al. CT Imaging Features of 2019 Novel Coronavirus (2019-nCoV). Radiology. 2020; (295): 202-207.

4. Wong HYF, Lam HYS, Fong AH-T, et al. Frequency and Distribution of Chest Radiographic Findings in Patients Positive for COVID-19. Radiology 2020; (296): E72-E78. 
5. Haseli S, Khalili N, Bakhshayeshkaram M, Sanei Taheri M, Moharramzad Y. Lobar Distribution of COVID-19 Pneumonia Based on Chest Computed Tomography Findings; A Retrospective Study. Arch Acad Emerg Med. 2020; 8 .

6. Zu ZY, Jiang MD, Xu PP, et al. Coronavirus Disease 2019 (COVID-19): A Perspective from China. Radiology. 2020; (296): E15-E25.

7. Asgharian B, Price O, Oberdörster G. A Modeling Study of the Effect of Gravity on Airflow Distribution and Particle Deposition in the Lung. Inhalation Toxicology. 2006; (18): 473-481.

8. Warren MA, Zhao Z, Koyama T, et al. Severity scoring of lung oedema on the chest radiograph is associated with clinical outcomes in ARDS. Thorax. 2018; (73): 840-846.

9. Smoleń A. Biostatystyka w badaniach medycznych i praktyce klinicznej. Polskie Archiwum Medecyny Wewnętrznej. 2016; (1): 3-20.
10. Ai T, Yang Z, Hou H, et al. Correlation of Chest CT and RT-PCR Testing for Coronavirus Disease 2019 (COVID-19) in China: A Report of 1014 Cases. Radiology. 2020; (296): E32-E40.

11. Pan F, Ye T, Sun P, et al. Time Course of Lung Changes at Chest CT during Recovery from Coronavirus Disease 2019 (COVID-19). Radiology. 2020; (295): 715-721.

12. Bettinelli D, Kays C, Bailliart O, et al. Effect of gravity on chest wall mechanics. J Appl Physiol. 2002; (92): 709-716.

13. Yang R, Li X, Liu H, et al. Chest CT Severity Score: An Imaging Tool for Assessing Severe COVID-19. Radiology Cardiothoracic Imaging. 2020; (2): e200047.

14. Bao C, Liu X, Zhang H, Li Y, Liu J. Coronavirus Disease 2019 (COVID-19) CT Findings: A Systematic Review and Meta-analysis. J Am College Radiol. 2020; (17): 701-709. 